\title{
AP Deployment Research Based on Physical Distance and Channel Isolation
}

\author{
Dangui Yan, ${ }^{1}$ Chengchang Zhang, ${ }^{2}$ Honghua Liao, ${ }^{3}$ Lisheng Yang, ${ }^{2}$ \\ Ping $\mathrm{Li}^{2}{ }^{2}$ and Guogang Yang $^{2}$ \\ ${ }^{1}$ College of Mathematics and Physics, Chongqing University of Post and Telecommunications, Chongqing 400086, China \\ ${ }^{2}$ College of Communication Engineering, Chongqing University, Chongqing 400030, China \\ ${ }^{3}$ School of Information, Hubei University for Nationalities, Enshi 445000, China
}

Correspondence should be addressed to Dangui Yan; yandg@cqupt.edu.cn

Received 5 January 2014; Revised 26 February 2014; Accepted 26 February 2014; Published 31 March 2014

Academic Editor: Hamid Reza Karimi

Copyright (C) 2014 Dangui Yan et al. This is an open access article distributed under the Creative Commons Attribution License, which permits unrestricted use, distribution, and reproduction in any medium, provided the original work is properly cited.

\begin{abstract}
Aiming at the problem of inefficiency of wireless local area networks (WLAN) access point (AP) deployment in urban environment, a new algorithm for AP deployment based on physical distance and channel isolation (DPDCI) is proposed. First, it detects the position information of deployed APs and then calculates the interference penalty factor combined with physical distance and channel isolation, and finally gets the optimal location and channel of the new AP through the genetic algorithm. Comparing with NOOCA algorithm and NOFA-2 algorithm, the results of numerical simulation show that the new algorithm can minimize the mutual interference between basic service sets (BSS), can ensure the maximum of throughput based on quality of service (QoS) in BSS, and can effectively improve the system performance.
\end{abstract}

\section{Introduction}

With the development of information era, the growing importance of the wireless local area network (WLAN) becomes more and more obvious. Since the WLAN has the advantages of flexibility, simplicity, easy extension, and so on, it widely applies in hot places such as the markets, leisure clubs, and companies. However, there is no authoritative standards for commercial WLAN deployment and channel allocation and management standards, which leads to the current situation that each major telecom operator deploys its own WLAN equipment in the same hot spots in order to provide its own high-speed broadband multimedia business, respectively, which causes the repetitive construction of the coverage of the wireless access point (AP) and, at the same time, the large amount of channel interference in the limited frequency band due to the high-density deployment of AP. Therefore, how to effectively configure and optimize the AP channels becomes one of the leading problems to be solved for the large-scale commercial use of WLAN.
Aiming at AP channel interference problems, there have been some research results, most of which are mainly through the graph coloring [1], integer linear programming [2], and heuristic method [3] for allocating channels for APs in ISM (industrial, scientific, medical) band to make the whole interference minimum. Reference [4] uses the cognitive radio technology, combined with the service condition of the primary users' band, to allocate accessible primary users' channels for AP. However, the above algorithms improve the system throughput by minimizing interference, which neither considers the influence from different business on the throughput nor guarantees the quality of service (QoS) of the system. In order to ensure the QoS and fairness of different business in WLAN, based on Hsum algorithm [5], reference [6] introduces fairness index and puts forward the CAOTR (Channel Assignment based on the Order of Throughput Reduction) algorithm, but the complexity of the algorithm is higher. Reference [7] proposes the interference factor combining physical distance and channel isolation, but it is only suitable for the channel allocation of the fixedlocation AP and does not consider changing the deployment 


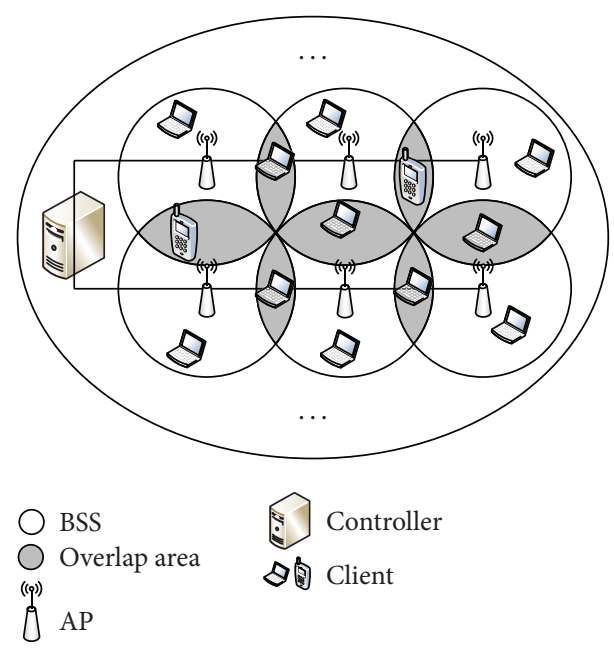

FIGURE 1: Work scene under the coexistence of high-density AP.

position of the AP. In [8], it gives a self-adapted algorithm based on neural network, which adapts retreat parameters in real time at Data Link Layer according to QoS request from Application Layer and channel state information from Physical Layer. In $[9,10]$, based on game theory algorithm, it seeks the optimal solution under the restricted condition of throughput, QoS, fairness, and so forth. However, [8-10] fail to involve the channel allocation of AP.

This paper considers synthetically the physical distance and channel isolation among APs and the position information of already deployed AP in detection area; a new algorithm for AP deployment based on physical distance and channel isolation (DPDCI) is proposed, which can effectively reduce interference and improve the system overall throughput.

\section{System Model}

2.1. Network Model. As shown in Figure 1, each AP and associated terminals in AP's communication range comprise a basic service set (BSS). In the BSS, terminals communicate with AP through the Media Access Control (MAC) protocol, while the communication between the APs is achieved by the IAPP [11], whose working principle is as follows: AP monitoring the adjacent AP beacon, including SNR information and the received signal strength and so on, and then AP will send its own information to the controller, including the number of terminals in the BSS. After the controller gets all the information from each AP, it will measure the overall throughput and allocate the channels. Because of the uncertainty of the AP position, it may cause the overlap on the range of BSS of different APs. In the overlap area, if channel allocation is not reasonable, it will cause interference problems when the terminals in the overlap area exchange data with the corresponding AP, especially when cochannel interference appears; it may even cause the communication interruption between client and its AP.
2.2. Interference Model. As shown in Figure 2, each AP owns two areas-one is communication area, and the other is interference area. Communication area is related to transmitter power and path loss and the receiver sensitivity corresponded to the real communication rate of the physical layer. While interference area is related to transmitter power, path loss and the receiver sensitivity corresponded to the minimum-supported communication rate of the physical layer. Apparently, the interference area is greater than or equal to communication area.

The communication radius (interference radius) is defined as [12]

$$
r=10^{\left(P_{t}-P_{r}-L_{0}\right) / 10 \alpha},
$$

where $P_{t}$ is transmitting power, $P_{r}$ is receiving power; $\alpha$ is channel attenuation factor, and $L_{0}$ is the channel attenuation with one meter distance from receiver. The communication radius $r_{1}$ is obtained based on the minimum effective received power and the interference radius $r_{2}$ is obtained according to the minimum received power under interference. To simplify the analysis, we assume that the communication radius and interference radius of each AP are $r_{1}$ and $r_{2}$, respectively.

2.2.1. Channel Interference Factor. Channel interference factor between APs is defined as follows;

$$
\phi\left(c_{i}, c_{j}\right)=\int_{-\infty}^{+\infty} S_{t}(f) S_{r}(f-\tau) d f,
$$

where $c_{i}$ is the channel index number allocated to $\mathrm{AP}_{i}, c_{i}$ is the channel index number allocated to $\mathrm{AP}_{j}, i, j \in\{1, \ldots, 11\}$, $S_{t}(f)$ is the transmitting power distribution of $\mathrm{AP}, S_{r}(f)$ is the receiving power distribution of $\mathrm{AP}$, and $\tau$ is off-set frequency.

In order to make it easy to analyze, the paper uses the IEEE $802.11 \mathrm{~b}$ as the WiFi to analyze, and the channel isolation is set to $5 \mathrm{MHz}$ and channel bandwidth is set to $22 \mathrm{MHz}$. The transmitting power distribution is defined as follows [7]:

$$
S_{t}(f)= \begin{cases}-50 \mathrm{~dB}, & \text { if }\left|f-f_{c}\right|>22 \mathrm{MHz}, \\ -30 \mathrm{~dB}, & \text { if } 11 \mathrm{MHz}<\left|f-f_{c}\right|<22 \mathrm{MHz}, \\ 0 \mathrm{~dB}, & \text { otherwise, }\end{cases}
$$

where $f_{c}$ is the central frequency.

It is obvious that $\phi\left(c_{i}, c_{j}\right) \in[0,1]$, and especially, $\phi\left(c_{i}, c_{j}\right)=$ 0 when two APs' channels are completely orthogonal.

2.2.2. Physical Distance Interference. When the distance between $\mathrm{AP}_{i}$ and $\mathrm{AP}_{j}$ is bigger than the summation of $r_{1}$ and $r_{2}$, its overlap area $A_{i j}$ is illustrated in Figure 2, where the distance between adjacent $\mathrm{AP}_{i}$ and $\mathrm{AP}_{j}$ is $d_{i j}$, namely, $A B$, the communication radius of $\mathrm{AP}_{i}$, namely, length of $A C$, is $r_{1}$, and the interference radius of $\mathrm{AP}_{j}$, namely, length of $B C$, is $r_{2}$; in $\triangle A B C$, we can obtain $\theta_{1}$ and $\theta_{2}$ according to the cosine theorem:

$$
\begin{array}{r}
\theta_{1}=\arccos \left(\frac{r_{1}^{2}+d_{i j}{ }^{2}-r_{2}^{2}}{2 r_{1} d_{i j}}\right) \\
\theta_{2}=\arccos \left(\frac{r_{2}^{2}+d_{i j}{ }^{2}-r_{1}^{2}}{2 r_{2} d_{i j}}\right),
\end{array}
$$


where $S_{1}$ is the difference value that corresponding sector area of $\theta_{1}$ in the $\mathrm{AP}_{i}$ communication area minuses the area of $\triangle A O C, S_{2}$ is the difference value that corresponding to sector area of $\theta_{2}$ in $\mathrm{AP}_{j}$ communication area minuses the area of $\triangle B O C$ :

$$
\begin{aligned}
S_{1} & =\frac{1}{2} \theta_{1} r_{1}^{2}-\frac{1}{2} \times r_{1} \sin \theta_{1} \times r_{1} \cos \theta_{1} \\
& =\frac{1}{2} \theta_{1} r_{1}^{2}-\frac{1}{4} r_{1}^{2} \sin \left(2 \theta_{1}\right) \\
S_{2} & =\frac{1}{2} \theta_{2} r_{2}^{2}-\frac{1}{2} \times r_{2} \sin \theta_{2} \times r_{2} \cos \theta_{2} \\
& =\frac{1}{2} \theta_{2} r_{2}^{2}-\frac{1}{4} r_{2}^{2} \sin \left(2 \theta_{2}\right) .
\end{aligned}
$$

According to symmetry, the expression of area $A_{i j}$ is

$$
\begin{aligned}
A_{i j} & =2\left(S_{1}+S_{2}\right) \\
& =\frac{1}{2} r_{1}^{2}\left[2 \theta_{1}-\sin \left(2 \theta_{1}\right)\right]+\frac{1}{2} r_{2}^{2}\left[2 \theta_{2}-\sin \left(2 \theta_{2}\right)\right] .
\end{aligned}
$$

Therefore, $A_{i j}$ can be expressed as

$$
A_{i j}=\left\{\begin{aligned}
\frac{1}{2} r_{1}^{2}\left[2 \theta_{1}-\sin \left(2 \theta_{1}\right)\right] & \\
+\frac{1}{2} r_{2}^{2}\left[2 \theta_{2}-\sin \left(2 \theta_{2}\right)\right], & d_{i j}<r_{1}+r_{2} \\
0, & d_{i j} \geq r_{1}+r_{2} .
\end{aligned}\right.
$$

2.2.3. Signal to Interference Ratio in BSS. To facilitate analysis, we make such assumptions: AP receives the same power from the client and client obeys Poisson distribution in communication area, and each client transmits data equiprobably. Therefore, ignoring the noise, the APi's SIR [13] can be expressed as

$$
\operatorname{SIR}_{i}=\frac{\pi r_{1}^{2}}{\sum_{j=1, j \neq i}^{N} \phi\left(c_{i}, c_{j}\right) A_{i j}} .
$$

To guarantee the communication quality, SIR must satisfy the following condition:

$$
\operatorname{SIR}_{i} \leq \gamma_{i}
$$

where $\gamma_{i}$ is the value of SIR threshold under $\mathrm{AP}_{i}$.

\section{Detection of Deployed APs}

In an urban environment, a lot of the APs are located in some place which unable to be observed directly and the physical distance information of AP is hard to be measured directly, so wireless locating method is introduced [14]. It is assumed that the position of the already deployed AP is $\left(x_{0}, y_{0}, z_{0}\right)$, the position of the $i$ th measuring point is $\left(x_{i}, y_{i}, z_{i}\right)$, the received signal intensity measured at the $i$ th measuring point is $r_{i}$, and the numerical relationship between AP and the measuring point can be expressed as

$$
\begin{aligned}
& {\left[\begin{array}{c}
-x_{1}^{2}-y_{1}^{2}-z_{1}^{2}+x_{k}^{2}+y_{k}^{2}+z_{k}^{2} \\
-x_{2}^{2}-y_{2}^{2}-z_{2}^{2}+x_{k}^{2}+y_{k}^{2}+z_{k}^{2} \\
\cdots \\
-x_{k-1}^{2}-y_{k-1}^{2}-z_{k-1}^{2}+x_{k}^{2}+y_{k}^{2}+z_{k}^{2}
\end{array}\right]} \\
& =\left[\begin{array}{cccc}
-2 x_{1}+2 x_{k} & -2 y_{1}+2 y_{k} & -2 z_{1}+2 z_{k} & \frac{a_{1}\left(r_{1}-r_{k}\right)}{5} \\
-2 x_{2}+2 x_{k} & -2 y_{2}+2 y_{k} & -2 z_{2}+2 z_{k} & \frac{a_{1}\left(r_{2}-r_{k}\right)}{5} \\
\ldots & \cdots & \cdots & \cdots \\
-2 x_{k-1}+2 x_{k} & -2 y_{k-1}+2 y_{k} & -2 z_{k-1}+2 z_{k} & \frac{a_{1}\left(r_{k-1}-r_{k}\right)}{5}
\end{array}\right]\left[\begin{array}{c}
x_{0} \\
y_{0} \\
z_{0} \\
\frac{1}{n}
\end{array}\right] .
\end{aligned}
$$

For simplicity, assume

$$
\left[\begin{array}{c}
-x_{1}^{2}-y_{1}^{2}-z_{1}^{2}+x_{k}^{2}+y_{k}^{2}+z_{k}^{2} \\
-x_{2}^{2}-y_{2}^{2}-z_{2}^{2}+x_{k}^{2}+y_{k}^{2}+z_{k}^{2} \\
\cdots \\
-x_{k-1}^{2}-y_{k-1}^{2}-z_{k-1}^{2}+x_{k}^{2}+y_{k}^{2}+z_{k}^{2}
\end{array}\right]=a
$$




$$
\left[\begin{array}{cccc}
-2 x_{1}+2 x_{k} & -2 y_{1}+2 y_{k} & -2 z_{1}+2 z_{k} & \frac{a_{1}\left(r_{1}-r_{k}\right)}{5} \\
-2 x_{2}+2 x_{k} & -2 y_{2}+2 y_{k} & -2 z_{2}+2 z_{k} & \frac{a_{1}\left(r_{2}-r_{k}\right)}{5} \\
\ldots & \cdots & \cdots & \cdots \\
-2 x_{k-1}+2 x_{k} & -2 y_{k-1}+2 y_{k} & -2 z_{k-1}+2 z_{k} & \frac{a_{1}\left(r_{k-1}-r_{k}\right)}{5}
\end{array}\right]=B .
$$

According to the Least-Square Estimation, we can obtain

$$
\left[\begin{array}{c}
x_{0} \\
y_{0} \\
z_{0} \\
\frac{1}{n}
\end{array}\right]=\left(B^{T} B\right)^{-1} B^{T} a \text {. }
$$

So, the position information of the deployed AP can be calculated and the position information of all APs in the area can be measured.

\section{Access Strategy in Authorized Frequency Band}

We define $s_{t}$ as the primary user channel state at time slot $t, s_{t}=0$ indicates that the primary user's channel is busy, $s_{t}=1$ stands for availability of primary user's channel, $\theta_{0}=\operatorname{Pr}\left\{s_{t}=0\right\}$ stands for busy probability of primary user's channel, and $\theta_{1}=\operatorname{Pr}\left\{s_{t}=1\right\}$ stands for available probability of primary user's channel. $s_{t}^{\prime}$ stands for the detection result of primary user's channel from secondary user, $s_{t}^{\prime}=0$ stands for that the detection of primary user's channel being busy, $s_{t}^{\prime}=1$ stands for that the detection of primary user's channel being available, $\pi_{0}=\operatorname{Pr}\left\{s_{t}^{\prime}=0\right\}$ stands for probability that the detection of primary user's channel is busy, and $\pi_{1}=\operatorname{Pr}\left\{s_{t}^{\prime}=1\right\}$ stands for probability that the detection of primary user's channel is available. The accuracy of the secondary user detection result is mainly expressed with detection probability $p_{\mathrm{de}}\left(p_{\mathrm{de}}=\operatorname{Pr}\left\{s_{t}^{\prime}=0 \mid s_{t}=0\right\}\right)$ and false alarm probability $p_{\mathrm{fa}}\left(p_{\mathrm{fa}}=\operatorname{Pr}\left\{s_{t}^{\prime}=0 \mid s_{t}=1\right\}\right)$. The access strategy of authorized frequency band can be expressed as [15]

$$
\begin{array}{ll}
\max _{\left\{r_{j}, x_{j}\right\}} & \sum_{j=0,1} \pi_{j} r_{j} \operatorname{Pr}\left\{r_{j} \leq \log _{2}\left[1+\frac{x_{j} g_{s s}}{\left(1-z_{j}\right) Y g_{p s}+n}\right]\right\} \\
\text { s.t. } & \sum_{j=0,1} \pi_{j} x_{j} \leq \Gamma_{1} \\
& \sum_{j=0,1} \pi_{j} \alpha_{0 j} x_{j} g_{s p} \leq \Gamma_{2},
\end{array}
$$

where $r_{j}$ and $x_{j}$ stand for the secondary user's state when channel state of authorized frequency band is $j . g_{s s}$ is the channel power gain between the secondary user's transmitter and receiver. $g_{p s}$ is the gain between the primary user's transmitter and secondary user's receiver. $g_{s p}$ is the gain between the secondary user's transmitter and primary user's receiver. $z_{j}=0$ stands for the busy state of authorized frequency channel. $z_{j}=1$ stands for the available state of authorized frequency channel. $\alpha_{i j}$ is the situation that, after authorization, the real state of frequency band is $i$ and the detected result is the posterior probability of $j . \Gamma_{1}$ is the maximum power of secondary user. $\Gamma_{2}$ is the interference threshold of the primary user corresponding to secondary user.

\section{The GA-Based AP Deployment Algorithm}

In order to solve the optimal solution of the target channel allocation function reasonably, we introduce genetic algorithm [16] to solve the optimal channel allocation problem of AP.

Genetic algorithm is viewed as a classic bionic algorithm, which imitates the selection process of the biological nature and selects the more adaptive individuals to reproduce with the rule of the survival of the fittest in order to form a new solution space through crossover and mutation and finally get the optimal solution of the problem. Each kind of AP deployment is viewed as an individual, and a limited kind of AP deployment constitutes a population.

5.1. Fitness Function. In order to obtain the optimal solution, we first build the fitness function. Fitness function reflects how adaptively an individual responds to the restricted conditions, and individuals with larger function values adapt better.

Assuming the positions of $\mathrm{AP}_{i}$ and $\mathrm{AP}_{j}$ are $\left(x_{i}, y_{i}, z_{i}\right)$ and $\left(x_{j}, y_{j}, z_{j}\right)$, respectively, so the distance between $\mathrm{AP}_{i}$ and $\mathrm{AP}_{j}$ is

$$
d_{i j}=\sqrt{\left(x_{i}-x_{j}\right)^{2}+\left(y_{i}-y_{j}\right)^{2}+\left(z_{i}-z_{j}\right)^{2}} .
$$

$I_{c}$ is defined as the whole interference factor in the area, and $I_{c}(i, j)=I_{c}(j, i)$, so $I_{c}$ can be expressed as

$$
I_{c}=2 \sum_{i=1}^{N} \sum_{j=i+1}^{N} I_{c}(i, j),
$$

where $N$ is the number of APs in the area.

The system interference is minimized when the whole interference factor in the area is the minimum. To make the influence on the throughput of the whole area minimum, fitness function is defined as

$$
\text { fit }=\frac{N}{I_{c}} \text {. }
$$




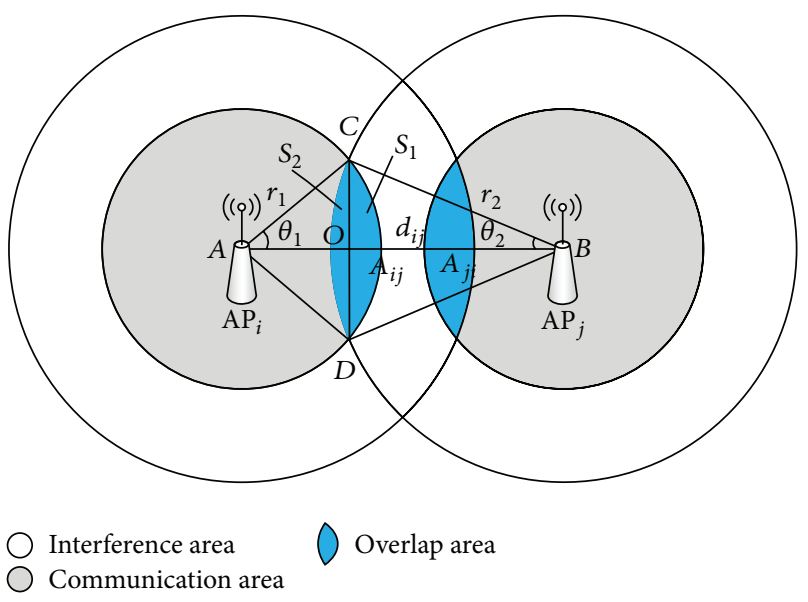

FIGURE 2: Diagram of interference model.

The probability that one individual is selected for crossover and mutation is defined as

$$
p_{i}=\frac{\text { fit }(i)}{\sum_{j=1}^{L} \text { fit }(j)},
$$

where $L$ is the number of individuals in the population.

5.2. The Pseudocode of the Algorithm. The pseudocode of the algorithm is as in Algorithm 1.

The input of the algorithm is the position information of deployed AP, the number of new AP, and information of AP-usable position. First, it allocates channels randomly for the AP and then allocates deployment point for the new APs using the deployable positions and generate a not completely same allocation result to constitutes a population, and calculate the adaptability function value of each individual in the population, and the number of the iteration is initialized as 0 . When the number of the iteration is smaller than the predefined maximum iteration number, calculating the selection probability according to (18), uses the Roulette method to select individual pair for hybrid processing, uses the Roulette method to select a single individual for mutation processing to get rid of individuals less adaptive individuals, and finally it refreshes the population and the number of iterations.

\section{Simulations}

To illustrate the performance of the algorithm, DPDCI algorithm is to be compared with the NOFA-2 algorithm [17] and the NOOCA algorithm [18].

The simulation parameters are shown in Table 1.

To estimate the interference of channel allocation algorithm over system performance, we define the average interference ratio $\eta$ of system user from channel interference as follows:

$$
\eta=\frac{1}{N} \sum_{i=1}^{N}\left(\frac{\sum_{j=1, j \neq i}^{N} A_{i j}}{\pi r_{1}^{2}}\right) .
$$

TABLE 1: Main parameters of numerical analysis.

\begin{tabular}{lc}
\hline Network topology size & $1 \mathrm{~km} \times 1 \mathrm{~km}$ \\
Transmit power of primary user's station $P_{\mathrm{pt}}$ & $43 \mathrm{dBm}$ \\
Received power of primary user's receiver $P_{\mathrm{pr}}$ & $-60 \mathrm{dBm}$ \\
Antenna gain of primary user's station $G_{\mathrm{pt}}$ & $15 \mathrm{dBi}$ \\
Antenna gain of primary user's receiver $G_{\mathrm{pr}}$ & $0 \mathrm{dBi}$ \\
Path loss parameter $\alpha$ of cognitive network & 4 \\
Interference noise power $\delta$ & $0.01 \mathrm{mw}$ \\
The reserved average transmit rate of primary user & $4 \mathrm{Mbps}$ \\
$C_{0}$ & $5 \mathrm{MHz}$ \\
Signal bandwidth $B$ & 11 \\
The total channel of primary user & 1 \\
Channel attenuation $L_{0}$ at $1 \mathrm{~m}$ & 15 \\
Available channel in authorized frequency band & 0.5 \\
Available probability of each channel in authorized & \\
frequency band & $100 \mathrm{~m}$ \\
Communication radius $r_{1}$ & $200 \mathrm{~m}$ \\
Interference radius $r_{2}$ &
\end{tabular}

The simulation results are as in Figure 3.

In Figure 3, the horizontal coordinate indicates the accessed number $n$ of AP and the vertical coordinate indicates the average interference of all the system users due to channel interference. At the first halves of the three algorithms, the tendencies of average interference are the same; this is due to the fact that the ISM band resource is relatively abundant under the situation that the number of AP is small. In particular, when the channel number is fewer than 3 , the system average interference is 0 since ISM band owns three orthogonal channels (1/6/11). When AP takes orthogonal channel, there has no mutually interference.

With the increase of the number of AP, the average interference of NOFA-2 is always higher than NOOCA and DPDCI since NOFA-2 only assigns channel in ISM band, while, in the lower part of ISM band, AP's SIR is over its threshold value; if AP number increases on this base, it will increase the channel interference and lead to the increase of average interference. Moreover, the average interference of NOOCA is bigger than that of DPDCI, which is because the fact that when the NOOCA algorithm assigns channel in hybrid band, it fails to consider that the AP in unauthorized channel cannot interfere with AP in ISM band, which results in suboptimal allocation result of certain ISM band channel.

The comparison of normalization throughput of the three algorithms is shown in Figure 4. NOOCA and DPDCI algorithms introduce authorized band channel. When the number of communication channels increases, it decreases the interference between APs. Therefore, they are better than NOFA-2 algorithm in normalization throughput. The DPDCI algorithm considers that the AP in unauthorized channel cannot interfere with AP in ISM band which makes the channel allocation in ISM band more optimal. Therefore, it has a higher normalization throughput over NOOCA algorithm. As the accessing client increases, the advantage of 
Input: a graph of APs with the No. of each AP

Output: an assigned channel for each AP

(1) Initial a population with different individual

(2) Calculated fitness for each individual

(3) Let gen $=0$

(4) While gen < GMAX do

(5) Assign a probability for each individual according to (18)

(6) Select many pair of the individual using the roulette method

(7) Crossover each pair of individual

(8) Select many individual using the roulette method

(9) Mutation each individual

(10) Calculated fitness for each individual

(11) Choose the right individual to form a new population

(12) gen $=$ gen ++

(13) End while

Algorithm 1

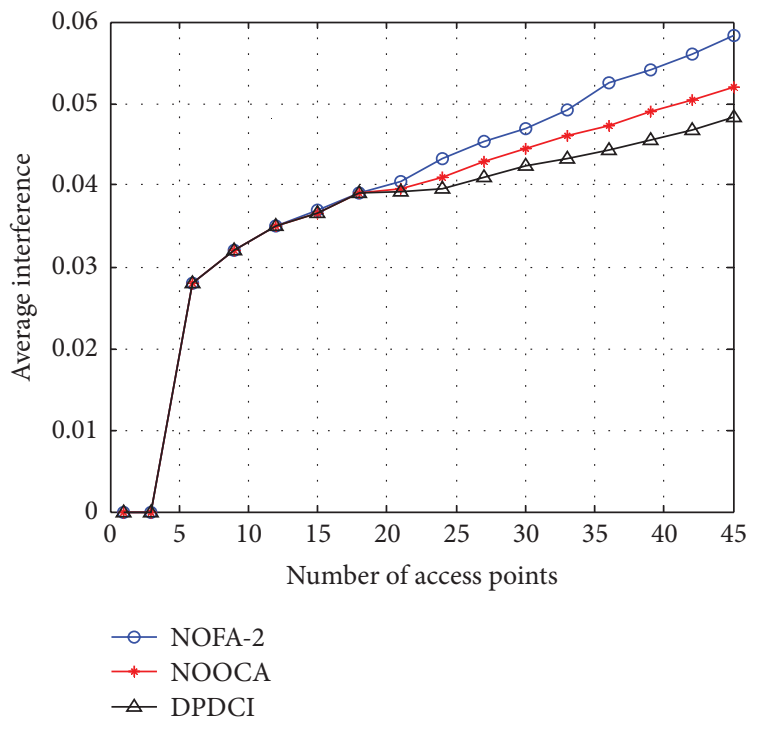

FIGURE 3: The comparison of average interference.

DPDCI and NOOCA algorithms is highlighted and they are obviously higher than NOFA-2 algorithm.

Figure 5 shows the normalized throughput of DPDCI algorithm in the cases of detecting already deployed APs and not detecting already deployed APs. Because detecting already deployed AP can effectively avoid the strong interference between new AP and already deployed AP due to too close physical distance, the optimization result of this case is better than the case of not detecting already deployed AP. From the whole point of view, the normalized throughput is improved about 3.7\%.

\section{Conclusion}

This paper gives a deep discussion on the new AP deployment in city area that has been deployed APs and uses the wireless locating method to detect the position of the already deployed

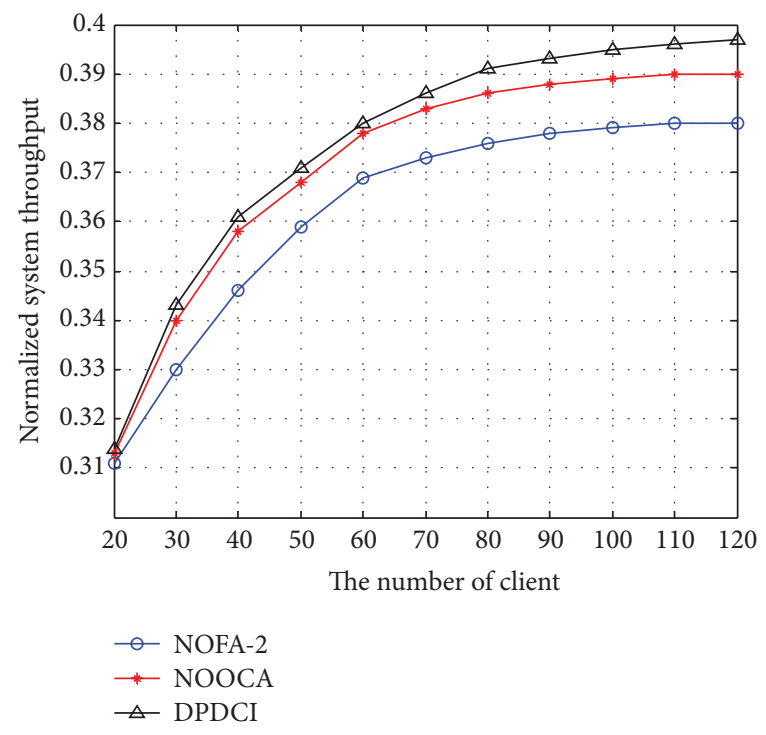

FIGURE 4: Comparison of normalization throughput.

APs that cannot be observed directly. Adopting genetic algorithm deploys new AP and allocates channels for AP; a new algorithm for AP deployment based on physical distance and channel isolation is proposed. The simulation results show that the new algorithm can minimize the mutual interference between BSS and ensure the maximum of throughput based on QoS in BSS and can also effectively improve the system performance. In order to achieve more practical oriented results, such as achieving dynamic configuration for the AP deployment, future work in the research will consider datadriven (measurements) framework [19-22].

\section{Conflict of Interests}

The authors declare that there is no conflict of interests regarding the publication of this paper. 


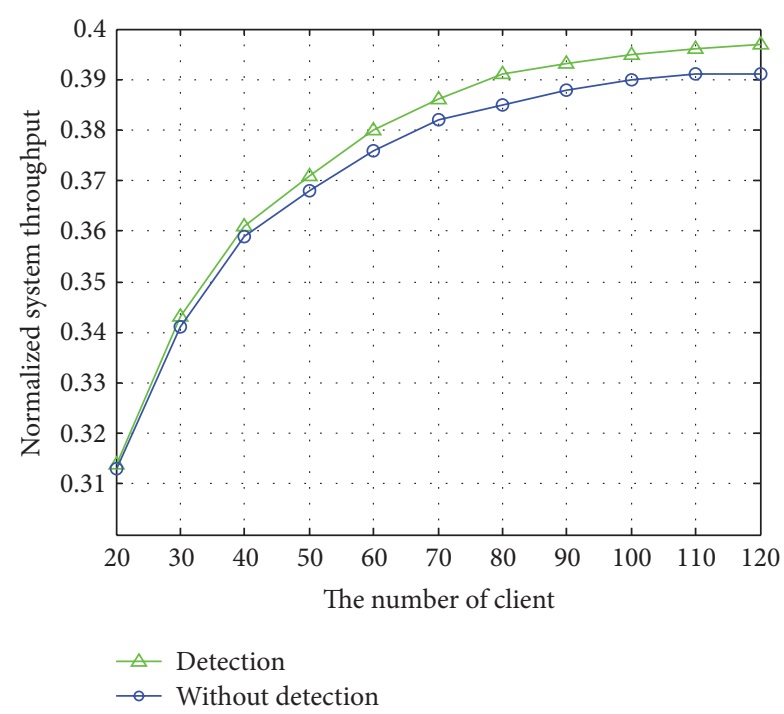

FIGURE 5: Comparison of DPDCI algorithm under detecting already deployed APs and not detecting already deployed APs.

\section{Acknowledgments}

The authors would like to thank the editors and the anonymous referees for their suggestions and comments. This work was supported in part by the National Natural Science Foundation of China (Grant no. 61263030), the Natural Science Foundation Project of CQCSTC (Grant no. 2010BB2240), and the Fundamental Research Funds for the Central Universities of China (Grants no. CDJZR12160018, no. CDJZR12160019).

\section{References}

[1] E. Amaldi, S. Bosio, F. Malucelli, and D. Yuan, "Solving nonlinear covering problems arising in WLAN design," Operations Research, vol. 59, no. 1, pp. 173-187, 2011.

[2] X.-N. Yue, C.-F. Wong, and S.-H. G. Chan, "CACAO: distributed client-assisted channel assignment optimization for uncoordinated WLANs," IEEE Transactions on Parallel and Distributed Systems, vol. 22, no. 9, pp. 1433-1440, 2011.

[3] A. Farsi, N. Achir, and K. Boussetta, "Heuristic approaches for access points deployment and Frequency Assignment in WLANs," in Proceedings of the Global Information Infrastructure Symposium (GIIS '11), pp. 1-7, IEEE Computer Society, Da Nang, Vietnam, August 2011.

[4] F. Novillo, M. Churchman, R. Ferrús, and R. Agusti, "A channel allocation algorithm for OSA-enabled IEEE 802.11 WLANs," in Proceedings of the 6th International Symposium on Wireless Communication Systems (ISWCS '09), pp. 468-472, Tuscany, Italy, September 2009.

[5] A. Mishra, S. Banerjee, and W. Arbaugh, "Weighted coloring based channel assignment for WLANs," Sigmobile Mobile Computing and Communications Review, vol. 3, no. 11, pp. 19-31, 2005.

[6] H. Zhang, H. Ji, and W.-D. Ge, "Channel assignment with fairness for multi-AP WLAN based on distributed coordination function," in Proceedings of the IEEE Wireless Communications and Networking Conference (WCNC '11), pp. 392-397, Cancun, Mexico, March 2011.
[7] Y. Cui, W. Li, and X. Cheng, "Partially overlapping channel assignment based on "node orthogonality" for 802.11 wireless networks," in Proceedings of the IEEE International Conference on Computer Communications (INFOCOM '11), pp. 361-365, Shanghai , China, April 2011.

[8] C. Wang, T. Lin, and J.-L. Chen, "A cross-layer adaptive algorithm for multimedia QoS fairness in WLAN environments using neural networks," IET Communications, vol. 1, no. 5, pp. 858-865, 2007.

[9] K.-L. Du, M. N. S. Swamy, and Q. Ni, "A dynamic spectrum access scheme for cognitive radio networks," in Proceedings of the Canadian Conference on Electrical and Computer Engineering (CCECE '09), pp. 450-454, IEEE Computer Society, St. John's, Canada, May 2009.

[10] M. R. Javan and A. R. Sharafat, "Efficient and distributed SINRbased joint resource allocation and base station assignment in wireless CDMA networks," IEEE Transactions on Communications, vol. 59, no. 12, pp. 3388-3399, 2011.

[11] M. Haidar, R. Akl, H. Al-Rizzo, and Y. Chan, "Channel assignment and load distribution in a power-managed WLAN," in Proceedings of the 18th Annual IEEE International Symposium on Personal, Indoor and Mobile Radio Communications (PIMRC '07), pp. 1-5, IEEE Computer Society, Athens, Greece, September 2007.

[12] F. Novillo, M. Churchman, R. Ferrús, and R. Agustí, "A channel allocation algorithm for OSA-enabled IEEE 802.11 WLANs," in Proceedings of the 6th International Symposium on Wireless Communication Systems (ISWCS '09), pp. 468-472, Tuscany, Italy, September 2009.

[13] T. Kwon and J.-W. Choi, "Multi-group random access resource allocation for M2M devices in multicell systems," IEEE Communications Letters, vol. 16, no. 6, pp. 834-837, 2012.

[14] J. Koo and H. Cha, "Localizing WiFi access points using signal strength," IEEE Communications Letters, vol. 15, no. 2, pp. 187189, 2011.

[15] W. Yuan, D. H. K. Tsang, L. Qian, and L. Meng, "Sensing based joint rate and power allocations for cognitive radio systems," IEEE Communications Letters, vol. 1, no. 2, pp. 113-116, 2012.

[16] Y. Yang and X.-J. Yu, "Cooperative coevolutionary genetic algorithm for digital IIR filter design," IEEE Transactions on Industrial Electronics, vol. 54, no. 3, pp. 1311-1318, 2007.

[17] W. El-Hajj and H. Alazemi, "Optimal frequency assignment for IEEE 802.11 wireless networks," Wireless Communications and Mobile Computing, vol. 9, no. 1, pp. 131-141, 2009.

[18] F. Novillo, M. Churchman, R. Ferrús, and R. Agustí, "A channel allocation algorithm for OSA-enabled IEEE 802.11 WLANs," in Proceedings of the 6th International Symposium on Wireless Communication Systems (ISWCS '09), pp. 468-472, Tuscany, Italy, September 2009.

[19] M. de la Sen, "On the characterization of hankel and toeplitz operators describing switched linear dynamic systems with point delays," Abstract and Applied Analysis, vol. 2009, Article ID 670314, 34 pages, 2009.

[20] H. Li, X. Jing, and H. R. Karimi, “Output-feedback-based $H_{\infty}$ control for vehicle suspension systems with control delay," IEEE Transactions on Industrial Electronics, vol. 1, no. 61, pp. 436-446, 2014.

[21] S. Yin, H. Luo, and S. X. Ding, "Real-time implementation of fault-tolerant control systems with performance optimization," IEEE Transactions on Industrial Electronics, vol. 5, no. 61, pp. 2402-2411, 2014.

[22] H. Li, X. Jing, H.-K. Lam, and P. Shi, "Fuzzy sampled-data control for uncertain vehicle suspension systems," IEEE Transactions on Cybernetics, no. 99, pp. 2168-2267, 2013. 


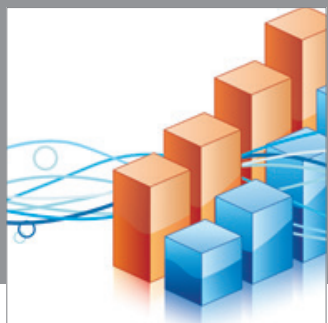

Advances in

Operations Research

mansans

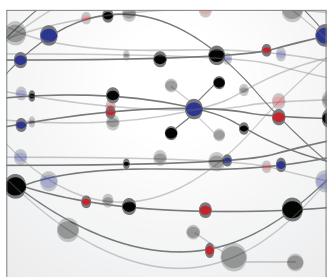

The Scientific World Journal
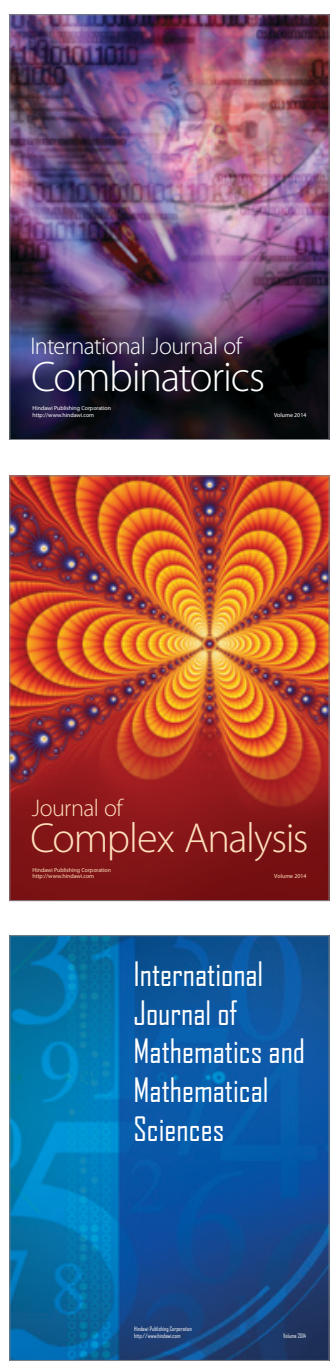
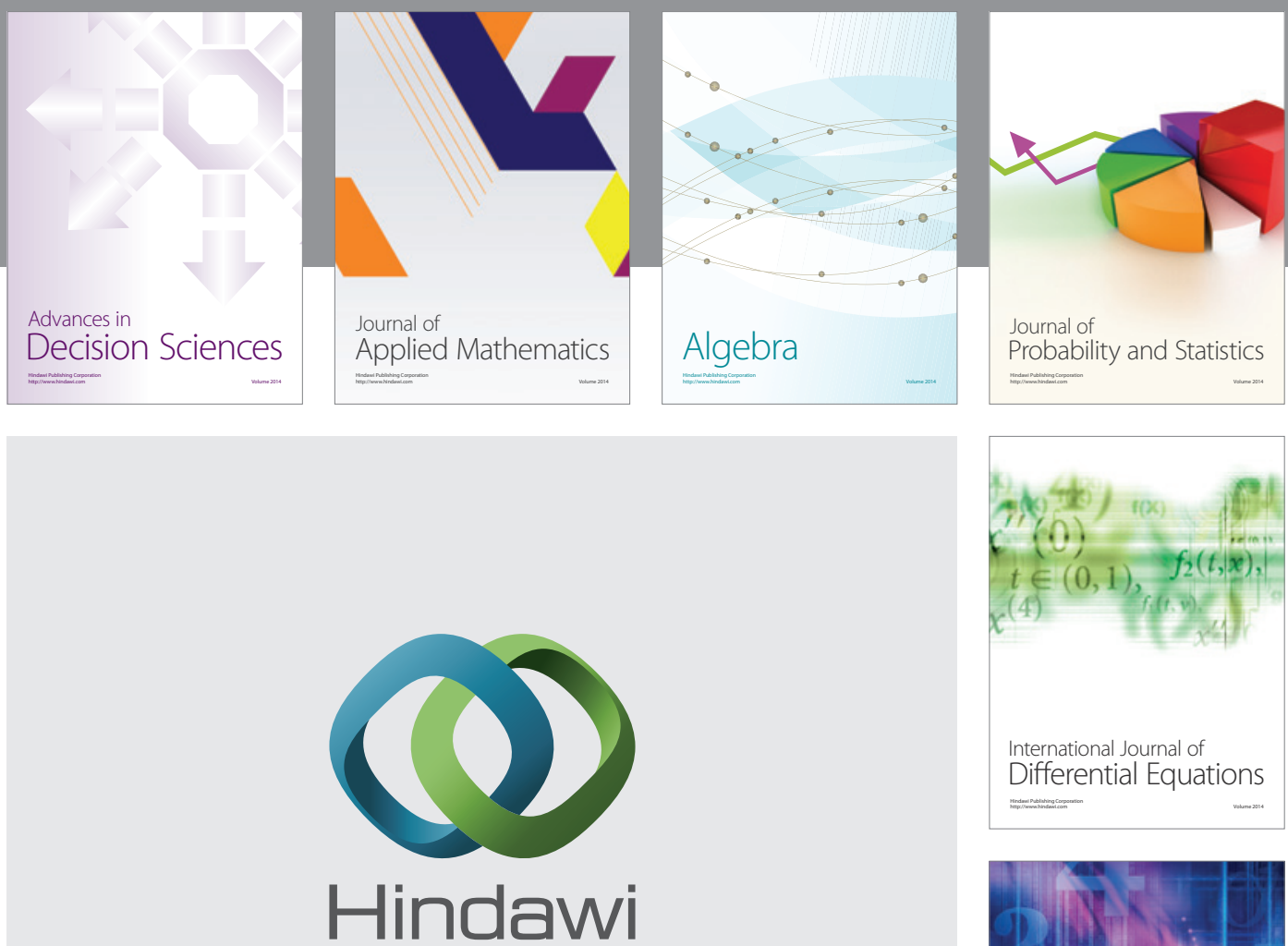

Submit your manuscripts at http://www.hindawi.com
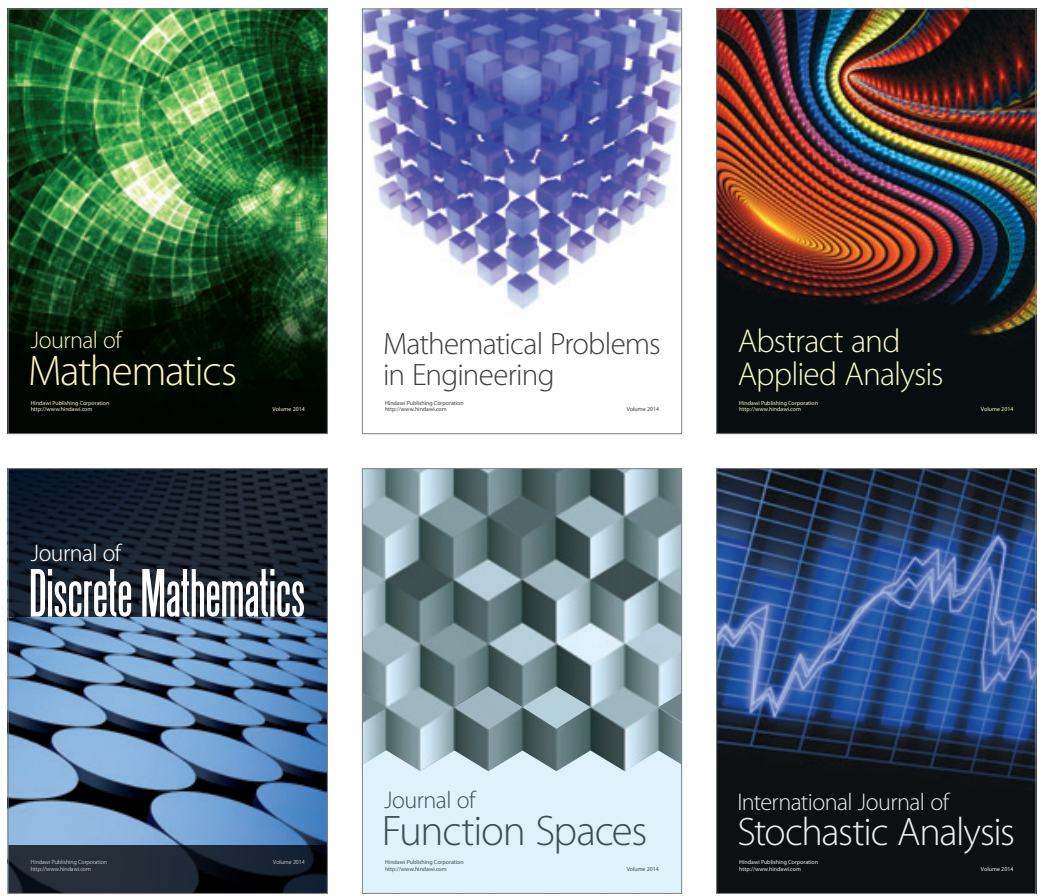

Journal of

Function Spaces

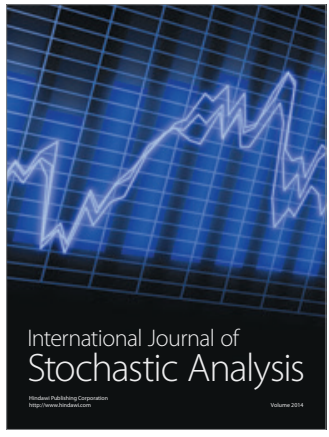

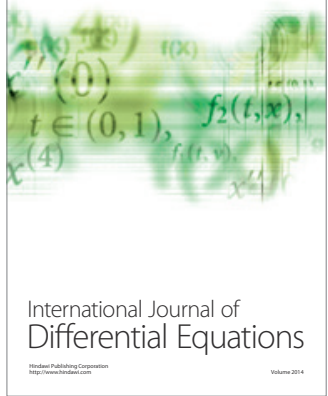
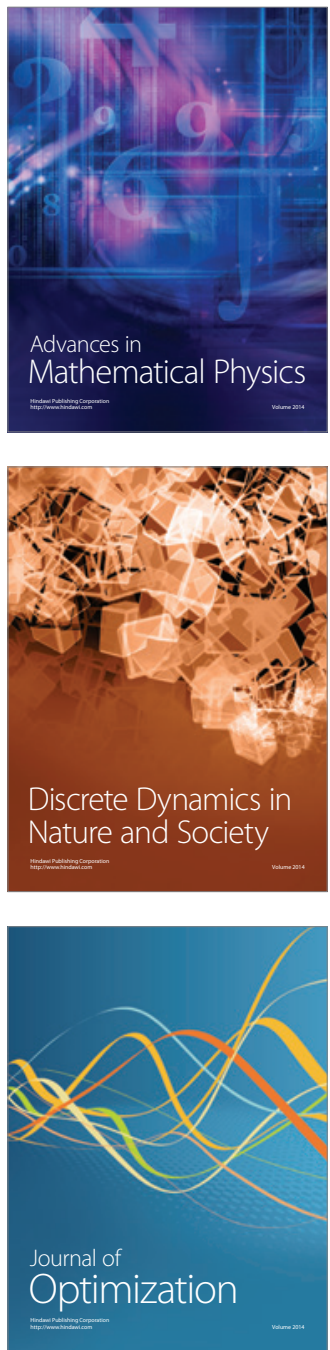